\title{
Erratum: Continuous wave superconducting radio frequency electron linac for nuclear physics research [Phys. Rev. Accel. Beams 19, 124801 (2016)]
}

\author{
Charles E. Reece® \\ (Received 18 October 2019; published 10 December 2019)
}

DOI: 10.1103/PhysRevAccelBeams.22.129901

Because additional reference material has recently become accessible, it may be helpful to amend the review of the evolution of SRF technology used in CEBAF by highlighting how some critical technical issues were successfully addressed during the establishment of CEBAF, when funding support for the construction of this innovative facility was yet vulnerable. In addition to the historical account of the founding of CEBAF by Catherine Westfall referenced in the original paper, Westfall also documented the prehistory of Jefferson Lab's SRF accelerating cavities, 1962 to 1985 [1], and the challenges associated specifically with Jefferson Lab's 1985 switch to superconducting accelerator technology [2]. The contingent nature of the choices leading to the unique tool that CEBAF is for nuclear physics makes for interesting reading.

It is also worth noting that the production of the original SRF cryomodules for CEBAF addressed in Sec. VIII was unprecedented in scale [3]. Quality assurance issues that were previously tolerable on a research or "one-off" scale required deliberate management to provide the reliability required for this first-in-class facility. In 1991, Hermann Grunder asked Fred Dylla to lead the cryomodule production effort. The immediate issue was assuring vacuum integrity of the CEBAF niobium cavity pair assemblies, each of which has 16 indium-wire sealed flange pairs that must be tight against superfluid helium. Under Dylla's leadership, a potential shutdown of the project was avoided when a path to a reliable beam line vacuum was established [4]. Subsequent to this experience, all SRF-based accelerator designs have used hard metal seal gaskets and avoid direct flange seals between superfluid helium and the beam line vacuum. Dylla also stimulated production quality improvements in several ancillary systems contributing to the successfully deployed CEBAF SRF cryomodules. Rapid refinement in 1991-1992 of designs and methods integral to the critical rf ceramic windows and cryogenic higherorder-mode absorbers, for example, enabled the project to proceed on schedule.

[1] C. Westfall, The Prehistory of Jefferson Lab's SRF Accelerating Cavities, 1962 to 1985 Jefferson Lab archives, 1997 (JLAB-PHY97-35), from https://misportal.jlab.org/ul/publications/downloadFile.cfm?pub_id=11132.

[2] C. Westfall, Jefferson Lab's 1985 Switch to Superconducting Accelerator Technology Jefferson Lab archives, 1996 (CEBAF-PR96-29), from https://misportal.jlab.org/ul/publications/downloadFile.cfm?pub_id=11131.

[3] H. F. Dylla, L. R. Doolittle, and J. F. Benesch, Operating experience with high beta superconducting RF cavities, in Proceedings of the 15th Particle Accelerator Conference, PAC-1993, Washington, DC, 1993 (IEEE, New York, 1993), p. 748, https://doi.org/ 10.1109/pac.1993.308784.

[4] H.F. Dylla et al. Vacuum Integrity of SRF Cavity Pair Assemblies JLab Technote, 1991 (CEBAF-TN-91-111). Available from https://jlabdoc.jlab.org/docushare/dsweb/Get/Document-202297/Vacuum\%20Integrity\%20of\%20SRF\%20Cavity\%20Pair $\% 20$ Assemblies.pdf.

Published by the American Physical Society under the terms of the Creative Commons Attribution 4.0 International license. Further distribution of this work must maintain attribution to the author(s) and the published articles title, journal citation, and DOI. 